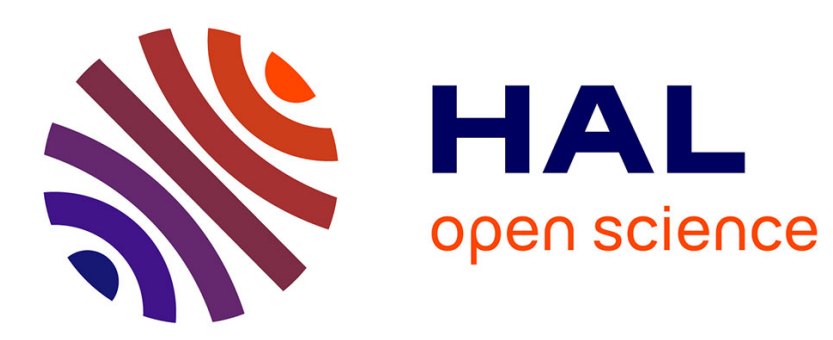

\title{
A counter-example to uniqueness in quasi-static elastic contact problems with small friction \\ Patrick Ballard
}

\section{To cite this version:}

Patrick Ballard. A counter-example to uniqueness in quasi-static elastic contact problems with small friction. International Journal of Engineering Science, 1999, 37 (2), pp.163-178. 10.1016/S00207225(98)00062-7 . hal-00111613

\section{HAL Id: hal-00111613 \\ https://hal.science/hal-00111613}

Submitted on 2 Apr 2018

HAL is a multi-disciplinary open access archive for the deposit and dissemination of scientific research documents, whether they are published or not. The documents may come from teaching and research institutions in France or abroad, or from public or private research centers.
L'archive ouverte pluridisciplinaire HAL, est destinée au dépôt et à la diffusion de documents scientifiques de niveau recherche, publiés ou non, émanant des établissements d'enseignement et de recherche français ou étrangers, des laboratoires publics ou privés.

\section{다)(1) $(5$}

Distributed under a Creative Commons Attribution - NonCommercial| 4.0 International 


\title{
A counter-example to uniqueness in quasi-static elastic contact problems with small friction
}

\author{
Patrick Ballard* \\ Laboratoire de Mécanique des Solides, Ecole Polytechnique, 91128 Palaiseau Cédex, France
}

It is often conjectured that the existence and uniqueness of solutions to the quasi-static Signorini problem with Coulomb friction should hold, provided that the friction coefficient is lower than a critical value. Recently, the existence of solutions to the quasi-static Signorini problem with non-local Coulomb friction was shown (M. Cocu, E. Pratt, M. Raous, Int. J. Engng. Sci. 34 (1996) 783-798) in functional spaces of type $W^{1, p}(0, T)$ and for a sufficiently low friction coefficient. In this paper, it is proved that uniqueness does not hold, in general, for an arbitrarily small friction coefficient.

\section{Introduction: the Signorini problem with Coulomb friction}

The problem of the equilibrium of a linear elastic body submitted to unfrictional unilateral contact conditions with a rigid obstacle was first considered by Signorini [1] and solved by Fichera [2]. Fichera's existence and uniqueness proof was followed immediately by general results on abstract variational inequalities [3]. These results allowed the solution of many nonlinear boundary problems [4-6].

From a mechanical point of view, the will to incorporate friction in Fichera's analysis of the Signorini problem rapidly emerged [7] and what is often called the Signorini problem with Coulomb friction began to be considered. This problem is not an equilibrium problem any more but an evolution problem. This problem faced great mathematical difficulties. For this reason, only an equilibrium problem (the so-called static one), obtained by a simplification of

* Tel.: 00-69-33-35-06; Fax: 00-69-33-30-26; e-mail: ballard@athena.polytechnique.fr. 
the equations, was first considered [4]. Having introduced a regularization (the non-local friction law), existence and uniqueness of the solution to the regularized static problem was shown by Duvaut [8-10] under a condition on the friction coefficient: it must be lower than a critical value. This result was followed by an existence result of the static problem without regularization and under a similar condition on the friction coefficient [11,12]. The results concerning the static problem allowed Klarbring to prove the existence and uniqueness of solutions of the rate problem associated with the regularized (by non-local friction law) quasistatic problem [13]. This result was still obtained under the condition on the friction coefficient. It is worth underlining that, as far as systems with a finite number of degrees of freedom (dof) are considered, the non-local regularized problem reduces to the unregularized one. To stress the importance of the condition on the friction coefficient, Klarbring [15] performed the complete analysis of the rate problem associated with a 2 dof quasi-static problem and exhibited explicitly the condition on the friction coefficient for which existence and uniqueness for the rate problem was achieved. He showed that as soon as this condition is violated, nonuniqueness of solution to the evolution problem may be observed. From a mechanical point of view, the non-uniqueness for great friction coefficient is attributed to the physical irrelevancy of the model, since inertia forces are neglected.

These results have led the community to conjecture that, provided the condition on the friction coefficient, existence and uniqueness of solutions to the quasi-static Signorini problem with Coulomb friction should hold. A first step towards this direction was accomplished recently by Cocu et al. [14] who proved the existence of solutions to the non-local regularized quasi-static problem in functional spaces of type $W^{1, p}(0, T)$.

In this paper, an $n$ dof problem is considered. A complete analysis of this problem is provided. For $n=2$, this problem reduces to Klarbring's one [15]. In this case, it is proved that, under Klarbring's condition, existence and uniqueness of the solution to the evolution problem is achieved in an appropriate functional framework (let us recall that Klarbring proved only the existence and uniqueness for the associated rate problem). For the case $n \geq 3$, existence is proved under a condition which generalizes Klarbring's one. A counter-example is constructed which shows that in this case, uniqueness does not hold, in general, for an arbitrarily small friction coefficient.

\section{Presentation of the problem and a statement of the results}

\subsection{Description of the problem and notations}

Let $n(n \geq 2)$ be an arbitrary integer (the interesting cases will be $n=2$ and $n=3)$. $\left(0, \mathbf{e}_{N}\right.$, $\left.\mathbf{e}_{T 1}, \ldots, \mathbf{e}_{T(n-1)}\right)$ is an orthonormal coordinate system in euclidean $\mathbb{R}^{n}$. A punctual particle, whose position at time $t$ is given by $\mathbf{U}(t)=U_{N}(t) \mathbf{e}_{N}+\mathbf{U}_{T}(t)$, is considered, where $\mathbf{U}_{T}(t)$ is the orthogonal projection of $\mathbf{U}_{T}(t)$ on the subspace of $\mathbb{R}^{n}$ spanned by $\left(\mathbf{e}_{T 1}, \mathbf{e}_{T 2}, \ldots, \mathbf{e}_{T(n-1)}\right)$. All inertia effects are neglected. The particle is "kept" by a system of linear springs (see Fig. 1) so that the force exerted by the springs on the particle is (after linearization) $-\mathbf{K} \mathbf{U}(t)$, where $\mathbf{K}$ is a symmetric positive definite matrix of order $n$ (the stiffness matrix). Reciprocally, if $\mathbf{K}$ is an arbitrary symmetric positive definite matrix, it always corresponds to such a system of springs. An external force $\mathbf{F}(t)$, varying with time, is also applied on the particle. Moreover, the particle 


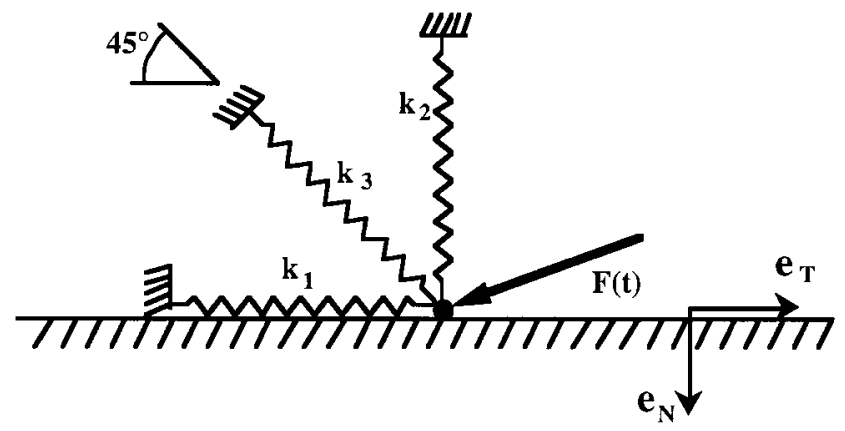

Fig. 1. Geometry and notations.

is supposed to obey to unilateral contact conditions with the half-space $U_{N} \geq 0$ and Coulomb friction law. Denoting by $\mathbf{R}(t)=R_{N}(t) \mathbf{e}_{N}+\mathbf{R}_{T}(t)$ the instantaneous reaction exerted by the obstacle on the particle, the equations of movement are given by:

$$
\begin{cases}\mathbf{K U}=\mathbf{F}+\mathbf{R} & \text { (equilibrium) } \\ U_{N} \leq 0, R_{N} \leq 0, U_{N} R_{N}=0 & \text { (unilateral contact) } \\ \forall \mathbf{V} \in \mathbb{R}^{n-1}, \mathbf{R}_{T}\left(\mathbf{V}-\dot{\mathbf{U}}_{T}\right)-\mu R_{N}\left(\|\mathbf{V}\|-\left\|\dot{\mathbf{U}}_{T}\right\|\right) \geq 0 & \text { (Coulomb friction) }\end{cases}
$$

where $\dot{\mathbf{U}}_{T}$ denotes the time derivative of $\mathbf{U}_{T}$ (supposed regular enough), $\mu$ the friction coefficient and $\|\cdot\|$ the usual norm in euclidean $\mathbb{R}^{n-1}$.

The variational formulation in part three of Eq. (1) of the Coulomb friction law was introduced by Duvaut \& Lions [4] and Moreau [16]. It is equivalent to the classical formulation. Note that Eq. (1) has formally the same structure as the equations of the Signorini problem with Coulomb friction for a continuum. The stiffness matrix $\mathbf{K}$ plays the role of the elastic energy bilinear form. In the sequel, $\mathbf{K}$ will be written under the form:

$$
\mathbf{K}=\left(\begin{array}{cc}
k_{N} & { }^{t} \mathbf{w} \\
\mathbf{w} & \mathbf{K}_{T}
\end{array}\right),
$$

where $\mathbf{w}$ is an element of $\mathbb{R}^{n-1}$ and $\mathbf{K}_{T}$ is a real matrix of order $n-1$. Note that the positive definiteness of $\mathbf{K}$ is equivalent to demand:

$$
k_{N}-{ }^{t} \mathbf{w} \cdot \mathbf{K}_{T}^{-1} \cdot \mathbf{w}>0, \quad \text { and } \quad \mathbf{K}_{T} \quad \text { symmetric positive definite. }
$$

\subsection{Statement of problem}

Let $T$ be an arbitrary strictly positive real number and $\mathbf{F}$ an arbitrary element of $W^{1, p}\left(0, T ; \mathbb{R}^{n}\right)(1 \leq p \leq \infty)$ such that $\mathbf{F}(0)=0$. The following problem is considered:

Problem $\mathcal{P}_{n}$ : find $\mathbf{U}$ and $\mathbf{R}$ in $W^{1, p}\left(0, T ; \mathbb{R}^{n}\right)$, such that:

$$
\begin{array}{ll}
\mathbf{U}(0)=0 & \\
\mathbf{K} \mathbf{U}(t)=\mathbf{F}(t)+\mathbf{R}(t), & \forall t \in[0, T] \\
U_{N}(t) \leq 0, R_{N}(t) \leq 0, U_{N}(t) R_{N}(t)=0, & \forall t \in[0, T] \\
\mathbf{R}_{T}(t)\left(\mathbf{V}-\dot{\mathbf{U}}_{T}(t)\right)-\mu R_{N}(t)\left(\|\mathbf{V}\|-\left\|\dot{\mathbf{U}}_{T}(t)\right\|\right) \geq 0, & \forall \mathbf{V} \in \mathbb{R}^{n-1} \text { and for a.a. } t \in[0, T] .
\end{array}
$$




\subsection{Statement of the results obtained from existing literature}

It is known from existing literature that:

- There exists a critical value $\mu_{\mathrm{c}}$ of the friction coefficient depending on the stiffness matrix $\mathbf{K}$, such that for a strictly lower friction coefficient $\mu$, there exists a unique solution to the rate problem associated with problem $\mathcal{P}_{\mathrm{n}}$ [13]. With the notations introduced above, the condition on the friction coefficient can be written:

$$
\mu<\frac{\lambda_{K}^{\min }}{\sqrt{k_{N}^{2}+\|w\|^{2}}},
$$

where $\lambda_{K}^{\min }$ denotes the lowest eigenvalue of the stifness matrix $\mathbf{K}$.

- There exists a critical value $\mu_{\mathrm{c}}$ of the friction coefficient depending on the stiffness matrix $\mathbf{K}$, such that for a strictly lower friction coefficient $\mu$, there exists at least one solution in $W^{1, p}\left(0, T ; \mathbb{R}^{n}\right) \times W^{1, p}\left(0, T ; \mathbb{R}^{n}\right)$ of problem $\mathcal{P}_{n}[14]$. The condition on the friction coefficient is expressed by condition (5).

- Under the condition:

$$
\mu<\frac{K_{T}}{|w|}
$$

the rate problem associated with problem $\mathcal{P}_{2}$ (note that in the case $n=2, w$ and $K_{T}$ reduce to real numbers) admits a unique solution in $\mathbb{R}^{2}$. If condition (6) is violated, then, the existence of the solution for the rate problem may be lost for certain load rates and uniqueness may also be lost for some other load rates [15]. In the case where condition (6) is violated, Klarbring gives also an example of a load history for which an infinite number of solutions to the evolution problem $\mathcal{P}_{2}$ are possible.

\subsection{Statement of the results obtained in this paper}

Denoting by $\lambda_{K_{T}}^{\min }$ the lowest eigenvalue of the matrix $\mathbf{K}_{T}$, and assuming that the following condition hold:

$$
\mu<\sqrt{\frac{\lambda_{K_{T}}^{\min }}{{ }^{t} \mathbf{w} \cdot \mathbf{K}_{T}^{-1} \cdot \mathbf{w}}},
$$

then, problem $\mathcal{P}_{n}$ admits at least one solution in $W^{1, p}\left(0, T ; \mathbb{R}^{n}\right) \times W^{1, p}\left(0, T ; \mathbb{R}^{n}\right)$. Moreover, if $n=2$ or $\mathbf{w}=\mathbf{0}$ this solution is unique and, if $n \geq 3$ and $\mathbf{w} \neq \mathbf{0}$ uniqueness does not hold, in general, whatever the strictly positive friction coefficient $\mu$ fulfilling condition (7) is. 


\subsection{Comments}

Problem $\mathcal{P}_{n}$ is a particular case of the general quasi-static Signorini problem with Coulomb friction considered in Ref. [14]. For this particular case, condition (7) on the friction coefficient for which existence holds is always less restrictive than condition (5) under which Cocu et al. proved the existence in the general case. This demonstrates that the Cocu et al. condition is not optimal. However, condition (7) is not optimal either in general. From a mechanical point of view, it is interesting to precise the optimal value of the critical friction coefficient, since it is related to a transition of the qualitative behaviour of the system. However, even for the simple system which is considered here, this optimal critical value cannot be explicitly expressed, in general, in terms of the matrix $\mathbf{K}$.

When the condition on the friction coefficient holds, uniqueness of the solution of Signorini problem with Coulomb friction is often conjectured since one knowns from Ref. [13] that the associated rate problem is well posed. The aim of this paper is to show that one has to answer by the negative to this conjecture, at least in the functional framework $W^{1, p}\left(0, T ; \mathbb{R}^{n}\right)$ in which the problem is usually considered.

\section{Proof of the announced results}

\subsection{Solution for the normal degree of freedom}

Proposition 1. Assuming that condition (7) holds the existence (respectively uniqueness) of a solution of problem $\mathcal{P}_{n}$ is equivalent to the existence (respectively uniqueness) of a solution of the following problem:

Problem $\mathcal{P}_{n-1}^{\prime}$ : find $\mathbf{U}_{T}$ and $\mathbf{R}_{T}$ in $W^{1, p}\left(0, T ; R^{n-1}\right)$, such that:

$$
\begin{cases}\mathbf{U}_{T}(0)=0 & \\ \mathbf{K}_{T} \mathbf{U}_{T}(t)=-U_{N}(t) \mathbf{w}+\mathbf{F}_{T}(t)+\mathbf{R}_{T}(t), & \forall t \in[0, T] \\ \mathbf{R}_{T}(t)\left[\mathbf{V}-\dot{\mathbf{U}}_{T}(t)\right]+\left[S(t)-\mu^{t} \mathbf{w} \cdot \mathbf{K}_{T}^{-1} \cdot \mathbf{R}_{T}(t)\right]\left(\|\mathbf{V}\|-\left\|\dot{\mathbf{U}}_{T}(t)\right\|\right) \geq 0, & \forall \mathbf{V} \in \mathbb{R}^{n-1} \text { and for a.a. } t \in[0, T]\end{cases}
$$

where:

$$
\begin{aligned}
U_{N}(t) & =-\frac{1}{k_{N}-{ }^{t} \mathbf{w} \cdot \mathbf{K}_{T}^{-1} \cdot \mathbf{w}}\left\langle F_{N}(t)-{ }^{t} \mathbf{w} \cdot \mathbf{K}_{T}^{-1} \cdot \mathbf{F}_{T}(t)\right\rangle^{-}, \\
S(t) & =\mu\left\langle F_{N}(t)-{ }^{t} \mathbf{w} \cdot \mathbf{K}_{T}^{-1} \cdot \mathbf{F}_{\mathrm{T}}(t)\right\rangle^{+} .
\end{aligned}
$$

If $x$ is a real number, $\langle x\rangle^{+}=\max (x, 0)$ and $(x)^{-}=\max (-x, 0)$ denote, respectively, the positive and negative parts of $x$.

Proof. First, one considers a solution $\mathbf{U}, \mathbf{R}$ of problem $\mathcal{P}_{n}$ and it is going to be proved that their tangential part $\mathbf{U}_{T}$ and $\mathbf{R}_{T}$ form a solution of problem $\mathcal{P}_{n-1}^{\prime}$. Let us define:

$$
\mathbf{F}^{*}(t)=\mathbf{K}^{-1} \mathbf{F}(t), \quad \mathbf{R}^{*}(t)=\mathbf{K}^{-1} \mathbf{R}(t) .
$$


It is clear that $\mathbf{F}^{*}$ and $\mathbf{R}^{*}$ belong to $W^{1, p}\left(0, T ; \mathbb{R}^{n}\right)$. The coordinate $R_{N}^{*}$ of $\mathbf{R}^{*}$ along $\mathbf{e}_{N}$ is:

$$
R_{N}^{*}(t)=\frac{R_{N}(t)-{ }^{t} \mathbf{w} \cdot \mathbf{K}_{T}^{-1} \cdot \mathbf{R}_{T}(t)}{k_{N}{ }^{t} \mathbf{w} \cdot \mathbf{K}_{T}^{-1} \cdot \mathbf{w}} .
$$

Moreover, part four of Eq. (4) implies:

$$
\forall t \in[0, T], \quad\left\|\mathbf{R}_{T}(t)\right\| \leq-\mu R_{N}(t) .
$$

Since $R_{N}(t)$ is negative, it is deduced from Eqs. (11) and (12):

$$
\forall t \in[0, T], \quad \frac{1+\mu\left\|\mathbf{K}_{T}^{-1} \cdot \mathbf{w}\right\|}{k_{N}-{ }^{t} \mathbf{w} \cdot \mathbf{K}_{T}^{-1} \cdot \mathbf{w}} R_{N}(t) \leq R_{N}^{*}(t) \leq \frac{1-\mu\left\|\mathbf{K}_{T}^{-1} \cdot \mathbf{w}\right\|}{k_{N}-{ }^{t} \mathbf{w} \cdot \mathbf{K}_{T}^{-1} \cdot \mathbf{w}} R_{N}(t) .
$$

It is readily seen that condition (7) implies:

$$
\mu\left\|\mathbf{K}_{T}^{-1} \cdot \mathbf{w}\right\|<1 .
$$

Multiplying each members of Eq. (13) by $U_{N}(t)$ which is negative, and using condition (14), one obtains, thanks to part three of Eq. (4):

$$
\forall t \in[0, T], \quad U_{N}(t) \leq 0, \quad R_{N}^{*}(t) \leq 0, \quad U_{N}(t) R_{N}^{*}(t)=0,
$$

This, coupled with

$$
\forall t \in[0, T], \quad U_{N}(t)=F_{N}^{*}(t)+R_{N}^{*}(t)
$$

leads to the following variational inequality:

$$
\forall t \in[0, T], \quad \forall V \in \mathbb{R}^{-}, \quad U_{N}(t)\left[V-U_{N}(t)\right] \geq F_{N}^{*}(t)\left[V-U_{N}(t)\right]
$$

The use of Lions-Stampacchia theorem [3] allows us to conclude that there exists a unique negative function $U_{N}(t)$ satisfying Eq. (17). Actually, one necessary has:

$$
\begin{aligned}
& U_{N}(t)=-\left\langle F_{N}^{*}(t)\right\rangle^{-}=-\frac{1}{k_{N}-{ }^{t} \mathbf{w} \cdot \mathbf{K}_{T}^{-1} \cdot \mathbf{w}}\left\langle F_{N}(t)-{ }^{t} \mathbf{w} \cdot \mathbf{K}_{T}^{-1} \cdot \mathbf{F}_{T}(t)\right\rangle^{-}, \\
& R_{N}^{*}(t)=-\left\langle F_{N}^{*}(t)\right\rangle^{+}=-\frac{1}{k_{N}{ }^{t}{ }^{t} \mathbf{w} \cdot \mathbf{K}_{T}^{-1} \cdot \mathbf{w}}\left\langle F_{N}(t)-{ }^{t} \mathbf{w} \cdot \mathbf{K}_{T}^{-1} \cdot \mathbf{F}_{T}(t)\right\rangle^{+} .
\end{aligned}
$$

A standard result ([6], Theorem A.1) shows that the functions $U_{N}(t)$ and $R_{N}^{*}(t)$ given by Eq. (18) belongs to $W^{1, p}(0, T ; \mathbb{R})$. Then, it is easily seen that the tangential parts $\mathbf{U}_{T}$ and $\mathbf{R}_{T}$ of $\mathbf{U}$ and $\mathbf{R}$ constitute a solution of problem $\mathcal{P}^{\prime}{ }_{n-1}$.

Reciprocally, let $\mathbf{U}_{T}$ and $\mathbf{R}_{T}$ be a solution of problem $\mathcal{P}_{n-1}^{\prime}$. From part three of Eq. (8), it is readily seen that, for all $t$ in $[0, T], S(t)-\mu^{t} \mathbf{w} \cdot \mathbf{K}_{T}^{-1} \cdot \mathbf{R}_{T}(t)$ is positive. Let us define:

$$
R_{N}(t)=-\frac{1}{\mu}\left[S(t)-\mu^{t} \mathbf{w} \cdot \mathbf{K}_{T}^{-1} \cdot \mathbf{R}_{T}(t)\right],
$$

which clearly belongs to $W^{1, p}(0, T ; \mathbb{R})$. Then, it is readily seen that the functions $\mathbf{U}$ and $\mathbf{R}$ 
whose tangential parts are $\mathbf{U}_{T}$ and $\mathbf{R}_{T}$ and normal parts are given respectively by Eqs. (9) and (19), constitute a solution of problem $\mathcal{P}_{n}$.

\subsection{Existence of a solution for problem $\mathcal{P}_{n-1}^{\prime}$}

Proposition 2. Assuming that condition (7) holds, problem $\mathcal{P}_{n-1}^{\prime}$ admits at least one solution in $W^{1, p}\left(0, T ; \mathbb{R}^{n-1}\right) \times W^{1, p}\left(0, T ; \mathbb{R}^{n-1}\right)$. Moreover, if $n=2$ or $\mathbf{w}=0$, this solution is unique.

Proof. First, notice that $\mathbf{K}_{T}$ defines a scalar product on $\mathbb{R}^{n-1}$, the associated norm being denoted by $\|\cdot\|_{K_{T}}$.

Let us introduce the following closed convex sets of $\mathbb{R}^{n-1}$ :

$$
\begin{aligned}
E(t) & =\left\{\mathbf{x} \in \mathbb{R}^{n-1} /\|\mathbf{x}\|+\mu^{t} \mathbf{w} \cdot \mathbf{K}^{-1} \cdot \mathbf{x} \leq S(t)\right\} \\
B(t, \mathbf{r}) & =\left\{\mathbf{x} \in \mathbb{R}^{n-1} /\|\mathbf{x}\| \leq\left\langle S(t)-\mu^{t} \mathbf{w} \cdot \mathbf{K}_{T}^{-1} \cdot \mathbf{r}\right\rangle^{+}\right\} .
\end{aligned}
$$

One obviously has:

$$
\forall \mathbf{r} \in E(t), \quad \mathbf{r} \in B(t, \mathbf{r})
$$

Owing to Eq. (14), $E(t)$ is a closed set bounded by an ellipsoid with one focus at the origin, and $B(t, \mathbf{r})$ is a ball centered at the origin. $E^{\circ}(t)$ and $\partial E(t)$ denoting as usual the interior and the boundary of $E(t)$, one defines the multivocal operator $A(t)$ by:

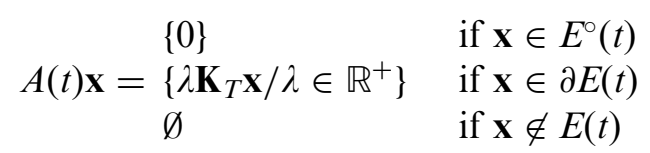

whenever $S(t) \neq 0$ [that is, $E(t) \neq\{0\}]$. When $S(t)=0$ [that is, $E(t)=\{0\}], A(t)$ is defined by:

$$
A(t) \mathbf{x}=\begin{array}{ll}
\mathbb{R}^{n-1} & \text { if } \mathbf{x} \in E(t)=\{0\} \\
\emptyset & \text { if } \mathbf{x} \notin E(t) .
\end{array}
$$

With these notations, problem $\mathcal{P}_{n-1}^{\prime}$ is easily seen to be equivalent to one or the other of the two following problems:

$$
\begin{aligned}
& \begin{cases}\mathbf{R}_{T}(0)=0 & \text { for a.a. } \mathrm{t} \in[0, T] \\
-\dot{\mathbf{R}}_{T}(t)-\dot{\mathbf{F}}_{T}(t) \in A(t) \cdot \mathbf{U}_{T}(t) & \\
\mathbf{K}_{T} \mathbf{U}_{T}(t)=-U_{N}(t) \mathbf{w}+\mathbf{F}_{T}(t)+\mathbf{R}_{T}(t), & \forall \mathrm{t} \in[0, T]\end{cases} \\
& \begin{cases}\mathbf{R}_{T}(0)=0 \\
-\dot{\mathbf{R}}_{T}(t)-\dot{\mathbf{F}}_{T}(t) \in \partial_{K_{T}^{-1}} I_{B\left[t, R_{T}(t)\right]} \cdot \mathbf{R}_{T}(t) & \text { for a.a. } \mathrm{t} \in[0, T] \\
\mathbf{K}_{T} \mathbf{U}_{T}(t)=-U_{N}(t) \mathbf{w}+\mathbf{F}_{T}(t)+\mathbf{R}_{T}(t), & \forall \mathrm{t} \in[0, T],\end{cases}
\end{aligned}
$$

where $\partial_{K_{T}^{-1}} I_{B\left[t, R_{T}(t)\right]}$ is the subdifferential of the indicatrix function of $B\left[t, \mathbf{R}_{T}(t)\right]$ for the scalar product $\mathbf{K}_{T}^{-1}$. To obtain formulations (24) and (25), we have used the fact that $S(t) \neq 0$ implies $\dot{U}_{N}(t)=0$. One may also obtain equivalent formulations by using unknown $\mathbf{U}_{T}(t)$ instead of $\mathbf{R}_{T}(t)$. For this, we define: 


$$
\begin{aligned}
K(t) & =\left\{\mathbf{x} \in \mathbb{R}^{n-1} / \mathbf{K}_{T} \mathbf{x}+U_{N}(t) \mathbf{w}-\mathbf{F}_{T}(t) \in E(t)\right\}, \\
C(t, \mathbf{u}) & =\left\{\mathbf{x} \in \mathbb{R}^{n-1} / \mathbf{K}_{T} \mathbf{x}+U_{N}(t) \mathbf{w}-\mathbf{F}_{T}(t) \in B\left[t, \mathbf{K}_{T} \mathbf{u}+U_{N}(t) \mathbf{w}-\mathbf{F}_{T}(t)\right]\right\} .
\end{aligned}
$$

One has:

$$
\forall \mathbf{u} \in K(t), \quad \mathbf{u} \in C(t, \mathbf{u}) .
$$

The multivocal operator $B(t)$ is defined by:

$$
\begin{aligned}
B(t) \mathbf{x}=\{0\} & \text { if } \mathbf{x} \in K^{\circ}(t) \\
\emptyset & \text { if } \mathbf{x} \in \partial K(t) \\
& \text { if } \mathbf{x} \notin K(t)
\end{aligned}
$$

whenever $S(t) \neq 0$. When $S(t)=0, B(t)$ is defined by:

$$
B(t) \mathbf{x}=\begin{array}{ll}
\mathbb{R}^{n-1} & \text { if } \mathbf{x} \in K(t) \\
\varnothing & \text { if } \mathbf{x} \notin K(t) .
\end{array}
$$

With these notations, problem $\mathcal{P}_{n-1}^{\prime}$ is easily seen to be equivalent to one or the other of the two following problems:

$$
\begin{aligned}
& \begin{cases}\mathbf{U}_{T}(0)=0 & \text { for a.a. } t \in[0, T] \\
-\dot{\mathbf{U}}_{T}(t) \in B(t) \cdot \mathbf{U}_{T}(t) & \forall t \in[0, T] \\
\mathbf{K}_{T} \mathbf{U}_{T}(t)=-U_{N}(t) \mathbf{w}+\mathbf{F}_{T}(t)+\mathbf{R}_{T}(t) & \forall t]\end{cases} \\
& \begin{cases}\mathbf{U}_{T}(0)=0 & \text { for a.a. } t \in[0, T], \\
-\dot{\mathbf{U}}_{T}(t) \in \partial_{K_{T}} I_{C\left[t, U_{T}(t)\right]} \cdot \mathbf{U}_{T}(t) & \forall t \in[0, T] \\
\mathbf{K}_{T} \mathbf{U}_{T}(t)=-U_{N}(t) \mathbf{w}+\mathbf{F}_{T}+\mathbf{R}_{T}(t), & \end{cases}
\end{aligned}
$$

where $\partial_{K_{T}} I_{C\left[t, U_{T}(t)\right]}$ is the subdifferential of the indicatrix function of $C\left[t, \mathbf{U}_{T}(t)\right]$ for the scalar product $\mathbf{K}_{T}$.

Note that, in general, part two of problem (30) is not monotone except for the cases $n=2$ or $\mathbf{w}=0$. For these cases, a standard argument gives uniqueness of a solution in $W^{1, p}\left(0, T ; \mathbb{R}^{n-1}\right)$. Now, it is going to be proved that problem (31) admits a solution by use of the Leray-Schauder fixed point theorem.

Let $\mathcal{H}[\cdot, \cdot]$ denote the Hausdorff distance associated with the norm $\|\cdot\|_{K_{T}}$ in $\mathbb{R}^{n-1}$ (for the definition and properties of the Hausdorff distance, see, for example, Ref. [6]).

Lemma 1. Let $t$ be an element of $[0, T]$ and $\mathbf{U}_{1}$ and $\mathbf{U}_{2}$ be in $K(t)$. One has:

$$
\mathcal{H}\left[C\left(t, \mathbf{U}_{1}\right), C\left(t, \mathbf{U}_{2}\right)\right] \leq \alpha\left\|\mathbf{U}_{1}-\mathbf{U}_{2}\right\|_{K_{T}}
$$

where:

$$
\alpha=\mu \sqrt{\frac{{ }^{t} \mathbf{W} \cdot \mathbf{K}_{T}^{-1} \cdot \mathbf{w}}{\lambda_{K_{T}}^{\min }}}<1 .
$$


Proof. Let $S_{1}, S_{2}$ be two positive real numbers and $C_{1}, C_{2}$ be the sets defined by:

$$
C_{i}=\left\{\mathbf{x} \in \mathbb{R}^{n-1} /\left\|\mathbf{K}_{T} \mathbf{x}\right\| \leq S_{i}\right\}, \quad i=1,2 .
$$

From the properties of the Hausdorff distance, one has:

$$
\mathcal{H}\left[C_{1}, C_{2}\right] \leq \mu\left|S_{2}-S_{1}\right| \operatorname{Max}\|\mathbf{u}\|_{K_{T}},
$$

where the maximum runs over the set of $\mathbf{u}$ such that $\left\|\mathbf{K}_{T} \mathbf{u}\right\|=1$. This maximum is readily seen to be $1 / \sqrt{ } \lambda_{K_{T}}^{\min }$ and inequality (35) is actually an equality. Hence,

$$
\mathcal{H}\left[C\left(t, \mathbf{U}_{1}\right), C\left(t, \mathbf{U}_{2}\right)\right]=\frac{\mu}{\sqrt{\lambda_{K_{T}}^{\min }}}\left|\left(\mathbf{U}_{2}-\mathbf{U}_{1}\right) \cdot \mathbf{w}\right| .
$$

The Cauchy-Schwartz inequality ends the proof of Lemma 1.

Lemma 2. There exist three positive real constants $A, B$ and $C$, such that, for every $\mathbf{U}_{T}$ in $W^{1, p}\left(0, T ; \mathbb{R}^{n-1}\right)$ :

$$
\begin{aligned}
\forall s, t \in[0, T] \quad \mathcal{H}\left[C \left(t, \mathbf{U}_{T}(t), C\left(s, \mathbf{U}_{T}(s)\right] \leq A\left\|\mathbf{F}_{T}(t)-\mathbf{F}_{T}(s)\right\|_{K_{T}}+B\left|U_{N}(t)-U_{N}(s)\right|\right.\right. \\
+C|S(t)-S(s)|+\alpha\left\|\mathbf{U}_{T}(t)-\mathbf{U}_{\mathrm{T}}(s)\right\|_{K_{T}} .
\end{aligned}
$$

Proof. The proof is straightforward by use of the following properties of the Hausdorff distance:

$$
\begin{aligned}
& \mathcal{H}[C+\{\mathbf{x}\}, C] \leq\|x\|_{K_{T}} \\
& \mathcal{H}\left[C_{1}, C_{3}\right] \leq \mathcal{H}\left[C_{1}, C_{2}\right]+\mathcal{H}\left[C_{2}, C_{3}\right]
\end{aligned}
$$

and inequality (35). One may choose:

$$
\begin{aligned}
& A=1+\frac{1}{\lambda_{K_{T}}^{\min }}, \\
& B=1+\|\mathbf{w}\|_{K_{T}}, \\
& C=\frac{1}{\sqrt{\lambda_{K_{T}}^{\min }}} .
\end{aligned}
$$

Now, one can prove proposition 2. The proof is adapted from that of Monteiro Marques [17] who considered a similar problem. Let $\mathcal{C}\left([0, T], \mathbb{R}^{n-1}\right)$ be the Banach space of the continuous functions from $[0, T]$ into $\mathbb{R}^{n-1}$, equipped with the uniform convergence norm (relative to the norm $\left.\|\cdot\|_{K_{T}}\right)$ denoted by $\|\cdot\|_{K_{T}}, \infty$. $\operatorname{Var}(\mathbf{f} ; a, b)$, where $\mathbf{f}$ belongs to $W^{1, p}\left(0, T ; \mathbb{R}^{n-1}\right)$ and $a, b$ to $[0, T]$ will be the classical variation of the function $\mathbf{f}$ over the interval $[a, b]$ in the sense of the norm $\|\cdot\|_{K_{T}}$. Let $\mathcal{K}$ be the subset of $\mathcal{C}\left([0, T], \mathbb{R}^{n-1}\right)$, constituted by the elements u such that $\mathbf{u}(0)=0$ and:

$$
\forall 0 \leq s \leq t \leq T,\|\mathbf{u}(t)-\mathbf{u}(s)\|_{K_{T}} \leq \frac{A}{1-\alpha} \operatorname{Var}\left(\mathbf{F}_{T} ; s, t\right)+\frac{B}{1-\alpha} \operatorname{Var}\left(U_{N} ; s, t\right)+\frac{C}{1-\alpha} \operatorname{Var}(S ; s, t) .
$$


It is obvious that $\mathcal{K}$ is non-empty, closed and convex. Moreover, it is equibounded (since $\mathbf{F}_{T}$, $U_{N}$ and $S$ have bounded variation over $[0, T]$ and equicontinuous by Eq. (40) (since $\mathbf{F}_{T}, U_{N}$ and $S$ are absolutely continuous); thus, by the Ascoli-Arzelà theorem, $\mathcal{K}$ is a compact subset of $\mathcal{C}\left([0, T], \mathbb{R}^{n-1}\right)$.

Note that if $\mathbf{u} \in \mathcal{K}$ then $\mathbf{u}$ is absolutely continuous [and even in $W^{1, p}\left(0, T ; \mathbb{R}^{n-1}\right)$ ] and by lemma $2, t \rightarrow C[t, \mathbf{u}(t)]$ is an absolutely continuous function. More precisely:

$$
\begin{aligned}
\operatorname{Var}(C ; s, t) \leq & A \operatorname{Var}\left(\mathbf{F}_{T} ; s, t\right)+B \operatorname{Var}\left(U_{N} ; s, t\right)+C \operatorname{Var}(S ; s, t) \\
& +\frac{\alpha A}{1-\alpha} \operatorname{Var}\left(\mathbf{F}_{T} ; s, t\right)+\frac{\alpha B}{1-\alpha} \operatorname{Var}\left(U_{N} ; s, t\right)+\frac{\alpha C}{1-\alpha} \operatorname{Var}(S ; s, t) \\
\leq & \frac{A}{1-\alpha} \operatorname{Var}\left(\mathbf{F}_{T} ; s, t\right)+\frac{B}{1-\alpha} \operatorname{Var}\left(U_{N} ; s, t\right)+\frac{C}{1-\alpha} \operatorname{Var}(S ; s, t) .
\end{aligned}
$$

Hence, by Moreau's results on sweeping processes [18], to every $\mathbf{u} \in \mathcal{K}$ one may associate $\Phi(\mathbf{u})=\mathbf{v}$, the unique absolutely continuous solution to the sweeping process:

$$
\left\{\begin{array}{l}
\mathbf{v}(0)=0 \\
-\dot{\mathbf{v}}(t) \in \partial_{K_{T}} I_{C[t, u(t)]} \cdot \mathbf{v}(t), \quad \text { for a.a. } t \in[0, T]
\end{array}\right.
$$

In order to apply the Leray-Schauder fixed point theorem, we have to prove that $\Phi(\mathbf{u}) \in \mathcal{K}$ and that $\mathbf{u} \rightarrow \Phi(\mathbf{u})$ is continuous in $\mathcal{K}$. In fact, $\Phi(\mathbf{u})$ is a continuous function, $\Phi(\mathbf{u})(0)=0$, and by Ref. [18] and Eq. (41), the following estimate holds:

$$
\begin{aligned}
\|\Phi(\mathbf{u})(t)-\Phi(\mathbf{u})(s)\|_{K_{T}} & \leq \operatorname{Var}(C ; s, t) \\
& \leq \frac{A}{1-\alpha} \operatorname{Var}\left(\mathbf{F}_{T} ; s, t\right)+\frac{B}{1-\alpha} \operatorname{Var}\left(U_{N} ; s, t\right)+\frac{C}{1-\alpha} \operatorname{Var}(S ; s, t),
\end{aligned}
$$

showing that $\Phi(\mathbf{u}) \in \mathcal{K}$. To prove that $\Phi$ is continuous, let $\mathbf{u}$ and $\mathbf{v}$ be two elements of $\mathcal{K}$. By the results of moreau on the dependence of solutions to sweeping processes on the data [18], we have:

$$
\| \Phi\left(\mathbf{u}(t)-\Phi(\mathbf{v})(t) \|_{K_{T}}^{2} \leq 2 m(t)\langle\operatorname{Var}[C(s, \mathbf{u}(s)] ; 0, t)+\operatorname{Var}\{C[s, \mathbf{v}(s)] ; 0, t\}\rangle,\right.
$$

where $m(t)$ is the least upper bound of $\mathcal{H}\{C[s, \mathbf{u}(s)], C[s, \mathbf{v}(s)]\}$ for $s \in[0, t]$. By lemma 1 , $m(t) \leq \alpha\|\mathbf{u}-\mathbf{v}\|_{K_{T}, \infty}$, which, by Eq. (41) gives:

$$
\|\Phi(\mathbf{u})-\Phi(\mathbf{v})\|_{K_{T}, \infty}^{2} \leq \frac{4 \alpha}{1-\alpha}\left[A \operatorname{Var}\left(\mathbf{F}_{T} ; 0, T\right)+B \operatorname{Var}\left(U_{N} ; 0, T\right)+C \operatorname{Var}(S ; 0, T)\right]\|\mathbf{u}-\mathbf{v}\|_{K_{T}, \infty}
$$

which shows the continuity of $\Phi$. 
From the Leray-Schauder theorem, there is a function $\mathbf{U}_{T} \in \mathcal{K}$ such that $\Phi\left(\mathbf{U}_{T}\right)=\mathbf{U}_{T}$. Clearly, $\mathbf{U}_{T} \in W^{1, p}\left(0, T ; \mathbb{R}^{n-1}\right)$ [because of Eq. (40)] and is a solution of problem (31) and, therefore of problem $\mathcal{P}_{n-1}^{\prime}$.

\subsection{A counter-example to uniqueness}

In this section, we consider problem $\mathcal{P}_{3}$ with the following particular form of the stiffness matrix:

$$
\mathbf{K}=\left(\begin{array}{lll}
1 & e & 0 \\
e & 1 & 0 \\
0 & 0 & 1
\end{array}\right)
$$

where $e$ is a given real number in the open interval ]0,1[. Given an arbitrary element $\mathbf{F}$ in $W^{1, p}\left(0, T ; \mathbb{R}^{n-1}\right)$ such that $\mathbf{F}(0)=0$, it is known from Section 3.1 and 3.2 that there exists at least one solution of this particular case of problem $\mathcal{P}_{3}$ provided that the following condition holds:

$$
\mu<\frac{1}{e} .
$$

Now, a load history $\mathbf{F}(t)$ in $W^{1, p}\left(0, T ; \mathbb{R}^{3}\right)$ is going to be constructed for an arbitrary $\mu$ in $] 0,1 /$ $e\left[\right.$. For this load history, two distinct solutions of problem $\mathcal{P}_{3}$ in $W^{1, \infty}\left(0, T ; \mathbb{R}^{3}\right)$ are going to be exhibited. This construction is not specific to the particular form of Eq. (46). Actually, such a construction can be performed whenever $n \geq 3$ and $\mathbf{w} \neq 0$. This particular case has been chosen for the sake of simplicity.

We start from the problem $\mathcal{P}_{2}^{\prime}$ associated with the particular form of Eq. (46) of the stiffness matrix. It has been seen that this problem can be written as:

$$
\begin{cases}\mathbf{R}_{T}(0)=0 & \\ -\dot{\mathbf{R}}_{T}(t)-\dot{\mathbf{F}}_{T} \in A(t) \cdot \mathbf{R}_{T}(t), & \text { for a.a } t \in[0, T] \\ \mathbf{U}_{T}(t)=-U_{N}(t) \mathbf{w}+\mathbf{F}_{T}(t)+\mathbf{R}_{T}(t), & \forall t \in[0, T],\end{cases}
$$

where $A(t)$ is, in this case the multivocal operator defined by:

$$
A(t) \mathbf{x}=\begin{array}{ll}
\{0\} & \text { if } \mathbf{x} \in E^{\circ}(t) \\
\mathbb{R}^{+} \mathbf{x} & \text { if } \mathbf{x} \in \partial E(t) \\
\emptyset & \text { if } \mathbf{x} \notin E(t),
\end{array}
$$

whenever $E(t) \neq\{0\}$. For $E(t)=\{0\}, A(t)$ is defined by:

$$
A(t) \mathbf{x}=\begin{array}{ll}
\mathbb{R}^{2}, & \text { if } \mathbf{x} \in E(t)=\{0\} \\
\emptyset, & \text { if } \mathbf{x} \notin E(t) .
\end{array}
$$

Let us recall that $E(t)$ is the closed convex set:

$$
E(t)=\left\{\left(x_{1}, x_{2}\right) \in \mathbb{R}^{2} / \sqrt{x_{1}^{2}+x_{2}^{2}}+e x_{1} \leq S(t)\right\} .
$$

We are now going to construct a function $S(t)$ in $W^{1, \infty}(0,1 ; \mathbb{R})$ such that $S(0)=0$ and a 
function $\mathbf{F}_{T}(t)$ in $W^{1, \infty}\left(0,1 ; \mathbb{R}^{2}\right)$, such that $\mathbf{F}_{T}(0)=0$ and two distinct solutions $\mathbf{R}_{T}^{a}(t)$ and $\mathbf{R}_{T}^{b}(t)$ of the associated problem (48). Then, as stated in proposition 1, these solutions will be used to construct two distinct solutions of problem $\mathcal{P}_{3}$ associated with the particular form of Eq. (46) of the stiffness matrix.

Let us begin to introduce the following notation:

$$
\beta=1+\frac{1-\sqrt{1-e^{2}}}{\sqrt{4-e^{2}}}
$$

$\beta$ is readily seen to be strictly greater than one.

Lemma 3. Let $T_{1}, T_{2}\left(T_{1}<T_{2}\right)$, be two real numbers. Let $S(t) .\left(T_{1} \leq t \leq T_{2}\right)$ be a constant function on $\left[T_{1}, T_{2}\right]$ with value $S(S>0)$. Then, there exists a function $\mathbf{F}^{\prime}{ }_{T}$ in $L^{\infty}\left(T_{1}, T_{2} ; \mathbb{R}^{2}\right)$ and two solutions $\mathbf{R}_{T}^{a}$ and $\mathbf{R}_{T}^{b}$ in $W^{1, \infty}\left(T_{1}, T_{2} ; \mathbb{R}^{2}\right)$ of problem:

$$
-\dot{\mathbf{R}}_{T}(t)-\mathbf{F}_{T}^{\prime}(t) \in A(t) \cdot \mathbf{R}_{T}(t), \quad \text { for a.a. } t \in\left[T_{1}, T_{2}\right]
$$

with initial conditions:

$$
\mathbf{R}_{T}^{a}\left(T_{1}\right)=(0,0) \quad \mathbf{R}_{T}^{b}\left(T_{1}\right)=\left(-\frac{S}{\beta} \frac{e}{1-e^{2}}, 0\right),
$$

and such that the following holds:

$$
\mathbf{R}_{T}^{a}\left(T_{2}\right)=(0,0) \quad \mathbf{R}_{T}^{b}\left(T_{2}\right)=\left(-S \frac{e}{1-e^{2}}, 0\right)
$$

Proof. It is easy to check that one may choose:

$$
\begin{array}{ll}
\mathbf{F}_{T}^{\prime}(t)=-\frac{5 S}{T_{2}-T_{1}}(0,1) & \forall t \in\left[T_{1}, T_{1}+\frac{T_{2}-T_{1}}{5}[\right. \\
\mathbf{F}_{T}^{\prime}(t)=-\frac{5 S}{T_{2}-T_{1}}\left(0, \frac{1-\sqrt{1-e^{2}}}{\beta \sqrt{1-e^{2}}}\right) & \forall t \in\left[T_{1}+\frac{T_{2}-T_{1}}{5}, T_{1}+\frac{2\left(T_{2}-T_{1}\right)}{5}[\right. \\
\mathbf{F}_{T}^{\prime}(t)=-\frac{5 S}{T_{2}-T_{1}}\left(-\frac{1}{2} \frac{e}{1-e^{2}}-1-\frac{1}{2} \sqrt{\frac{4-e^{2}}{1-e^{2}}}\right) & \forall t \in\left[T_{1}+\frac{2\left(T_{2}-T_{1}\right)}{5}, T_{1}+\frac{3\left(T_{2}-T_{1}\right)}{5}[\right. \\
\mathbf{F}_{T}^{\prime}(t)=-\frac{5 S}{T_{2}-T_{1}}\left(\frac{1-\beta}{\beta} \frac{e}{1-e^{2}}-\frac{1-\sqrt{1-e^{2}}}{\beta \sqrt{1-e^{2}}}\right) & \forall t \in\left[T_{1}+\frac{3\left(T_{2}-T_{1}\right)}{5}, T_{1}+\frac{4\left(T_{2}-T_{1}\right)}{5}[\right. \\
\mathbf{F}_{T}^{\prime}(t)=-\frac{5 S}{T_{2}-T_{1}}\left(\frac{1}{2} \frac{e}{1-e^{2}}, \frac{1}{2} \sqrt{\frac{4-e^{2}}{1-e^{2}}}\right) & \forall t \in\left[T_{1}+\frac{4\left(T_{2}-T_{1}\right)}{5}, T_{2}[\right.
\end{array}
$$


as well as the functions $\mathbf{R}_{T}^{a}(t)$ and $\mathbf{R}_{T}^{b}(t)$ defined by the linear interpolations of the values:

$$
\begin{aligned}
& \left\{\begin{array}{l}
\mathbf{R}_{T}^{a}\left(T_{1}\right)=(0,0) \\
\mathbf{R}_{T}^{b}\left(T_{1}\right)=S\left(-\frac{1}{\beta} \frac{e}{1-e^{2}}, 0\right)
\end{array}\right. \\
& \left\{\begin{array}{l}
\mathbf{R}_{T}^{a}\left(T_{1}+\frac{T_{2}-T_{1}}{5}\right)=S(0,1) \\
\mathbf{R}_{T}^{b}\left(T_{1}+\frac{T_{2}-T_{1}}{5}\right)=S\left(-\frac{1}{\beta} \frac{e}{1-e^{2}}, 1\right)
\end{array}\right. \\
& \left\{\begin{array}{l}
\mathbf{R}_{T}^{a}\left[T_{1}+\frac{2\left(T_{2}-T_{1}\right)}{5}\right]=S(0,1) \\
\mathbf{R}_{T}^{b}\left[T_{1}+\frac{2\left(T_{2}-T_{1}\right)}{5}\right]=S\left(-\frac{1}{\beta} \frac{e}{1-e^{2}}, 1+\frac{1-\sqrt{1-e^{2}}}{\beta \sqrt{1-e^{2}}}\right)
\end{array}\right. \\
& \left\{\begin{array}{l}
\mathbf{R}_{T}^{a}\left[T_{1}+\frac{3\left(T_{2}-T_{1}\right)}{5}\right]=S\left(-\frac{1}{2} \frac{e}{1-e^{2}},-\frac{1}{2} \sqrt{\frac{4-e^{2}}{1-e^{2}}}\right) \\
\mathbf{R}_{T}^{b}\left[T_{1}+\frac{3\left(T_{2}-T_{1}\right)}{5}\right]=S\left(-\left(\frac{1}{2}+\frac{1}{\beta}\right) \frac{e}{1-e^{2}},-\frac{1}{2} \sqrt{\frac{4-e^{2}}{1-e^{2}}}+\frac{1-\sqrt{1-e^{2}}}{\beta \sqrt{1-e^{2}}}\right)
\end{array}\right. \\
& \left\{\begin{array}{l}
\mathbf{R}_{T}^{a}\left[T_{1}+\frac{4\left(T_{2}-T_{1}\right)}{5}\right]=S\left(-\frac{1}{2} \frac{e}{1-e^{2}},-\frac{1}{2} \sqrt{\frac{4-e^{2}}{1-e^{2}}}\right) \\
\mathbf{R}_{T}^{b}\left[T_{1}+\frac{4\left(T_{2}-T_{1}\right)}{5}\right]=S\left(-\frac{3}{2} \frac{e}{1-e^{2}},-\frac{1}{2} \sqrt{\frac{4-e^{2}}{1-e^{2}}}\right)
\end{array}\right. \\
& \left\{\begin{array}{l}
\mathbf{R}_{T}^{a}\left(T_{2}\right)=(0,0) \\
\mathbf{R}_{T}^{b}\left(T_{2}\right)=S\left(-\frac{e}{1-e^{2}}, 0\right) .
\end{array}\right.
\end{aligned}
$$

The corresponding evolution of the segment $\left[\mathbf{R}_{T}^{a}, \mathbf{R}_{T}^{b}\right]$ is sketched on Fig. 2. The step function $\mathbf{F}_{T}^{\prime}(t)$ and the functions $\mathbf{R}_{T}^{a}(t)$ and $\mathbf{R}_{T}^{b}(t)$ defined by Eqs. (56) and (57) will be denoted in the sequel by $\mathcal{F}^{\prime}\left(T_{1}, T_{2}, S ; t\right), \mathcal{R}^{a}\left(T_{1}, T_{2}, S ; t\right)$ and $\mathcal{R}^{b}\left(T_{1}, T_{2}, S ; t\right)\left(T_{1} \leq t \leq T_{2}\right)$. It should be noted that there exists a constant $C(e)$ depending only on $e$ such that:

$$
\forall t \in\left[T_{1}, T_{2}\left[, \quad\left\|\mathcal{F}^{\prime}\left(T_{1}, T_{2}, S ; t\right)\right\| \leq \frac{S}{T_{2}-T_{1}} C(e) .\right.\right.
$$

Now, the intervals $\left[1 /\left(\beta^{m+1}\right), 1 / \beta^{m}\right.$, where $m$ is an arbitrary integer, define a partition of the interval ]0,1[. For every $m$ in $\mathbb{N}$, we define the function $S^{\prime}(t)$ on $\left[1 / \beta^{m+1}, 1 / \beta^{m}[\right.$ by:

$$
S^{\prime}(t)=\begin{aligned}
& 2 \text { if } t \in\left[\frac{1}{\beta^{m+1}}, \frac{1}{\beta^{m+1}} \frac{\beta+1}{2}[\right. \\
& 0 \quad \text { if } t \in\left[\frac{1}{\beta^{m+1}} \frac{\beta+1}{2}, \frac{1}{\beta^{m}}[\right.
\end{aligned}
$$

Hence, the function $S^{\prime}(t)$ is defined all over the interval ]0,1[, it is clearly measurable, bounded 

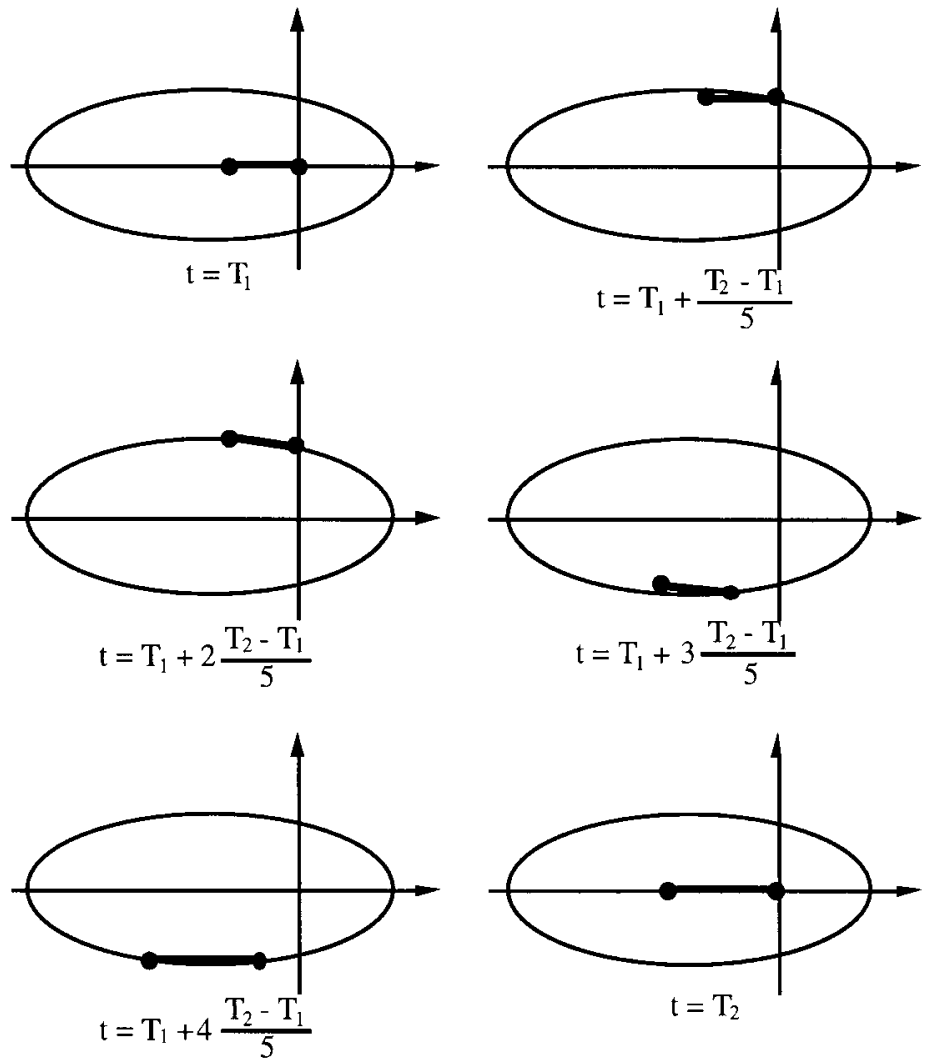

Fig. 2. Evolution of the two solutions constructed in lemma 3.

and therefore integrable. Let $S(t)$ be the absolutely continuous function defined by:

$$
S(t)=\int_{0}^{t} S^{\prime}(s) \mathrm{d} s
$$

The function $\mathrm{S}(t)$ is easily seen to belong to $W^{1, \infty}(0,1 ; \mathbb{R})$, to be positive and such that $S(0)=0$. Moreover, one has:

$$
\forall m \in \mathbb{N}, \quad \forall t \in\left[\frac{1}{\beta^{m+1}} \frac{\beta+1}{2}, \frac{1}{\beta^{m}}\right], \quad S(t)=\frac{1}{\beta^{m}} .
$$

Similarly, we define the function $\mathbf{F}_{T}^{\prime}(t)$ on the interval $\left[1 / \beta^{m+1}, 1 / \beta^{m}[\right.$ by:

$$
\mathbf{F}_{T}^{\prime}(t)=\begin{array}{ll}
0 & \text { if } t \in\left[\frac{1}{\beta^{m+1}}, \frac{1}{\beta^{m+1}} \frac{\beta+1}{2}[\right. \\
\mathcal{F}^{\prime}\left(\frac{1}{\beta^{m+1}} \frac{\beta+1}{2}, \frac{1}{\beta^{m}}, \frac{1}{\beta^{m}} ; t\right) & \text { if } t \in\left[\frac{1}{\beta^{m+1}} \frac{\beta+1}{2}, \frac{1}{\beta^{m}}[\right.
\end{array}
$$


The function $\mathbf{F}_{T}^{\prime}(t)$ is defined all over ]0,1[. From Eq. (58), it is bounded:

$$
\forall t \in] 0,1], \quad\left\|\mathbf{F}_{T}^{\prime}(t)\right\| \leq \frac{2 \beta}{\beta-1} C(e) .
$$

Since $\mathbf{F}_{T}^{\prime}(t)$ is clearly measurable, it is integrable and $\mathbf{F}_{T}(t)$ will be defined by:

$$
\mathbf{F}_{T}(t)=\int_{0}^{t} \mathbf{F}_{T}^{\prime}(s) \mathrm{d} s
$$

$\mathbf{F}_{T}(t)$ clearly belongs to $W^{1, \infty}\left(0,1 ; \mathbb{R}^{2}\right)$ and is such that $\mathbf{F}_{\boldsymbol{T}}(0)=0$, and $\dot{\mathbf{F}}_{\boldsymbol{T}}(\boldsymbol{t})=\mathbf{F}_{T}^{\prime}(t)$ almost everywhere in $[0,1]$. Finally, $\mathbf{R}_{T}^{a}(t)$ and $\mathbf{R}_{T}^{b}(t)$ are defined by:

$$
\begin{aligned}
& (0,0) \\
& \text { if } t \in\left[\frac{1}{\beta^{m+1}}, \frac{1}{\beta^{m+1}} \frac{\beta+1}{2}[\right. \\
& R_{T}^{a}= \\
& \mathcal{R}^{a}\left(\frac{1}{\beta^{m+1}} \frac{\beta+1}{2}, \frac{1}{\beta^{m}}, \frac{1}{\beta^{m}} ; t\right) \quad \text { if } t \in\left[\frac{1}{\beta^{m+1}} \frac{\beta+1}{2}, \frac{1}{\beta^{m}}[\right.
\end{aligned}
$$

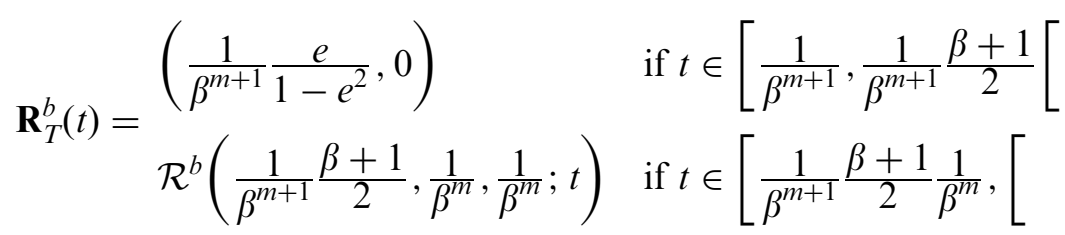

where $\mathcal{R}^{a}\left(T_{1}, T_{2}, S ; t\right)$ and $\mathcal{R}^{b}\left(T_{1}, T_{2}, S ; t\right)$ are the functions defined by lemma $3 . \mathbf{R}_{T}^{a}(t)$ and $\mathbf{R}_{T}^{b}(t)$ are readily seen to belong to $W^{1, \infty}\left(0,1 ; \mathbb{R}^{2}\right)$. Moreover, one checks that $\mathbf{R}_{T}^{a}(0)=\mathbf{R}_{T}^{b}(0)=0$ and also that one has:

$$
-\dot{\mathbf{R}}_{T}(t)-\dot{\mathbf{F}}_{T}(t) \in A(t) \cdot \mathbf{R}_{T}(t) \text { for a.a. } \in[0,1]
$$

where $\mathbf{R}_{T}(t)$ may be either $\mathbf{R}_{T}^{a}(t)$ or $\mathbf{R}_{T}^{b}(t)$. $\mathbf{R}_{T}^{a}$ and $\mathbf{R}_{T}^{b}$ lead to two solutions of problem $\mathcal{P}_{2}^{\prime}$ with the particular form of Eq. (46) of the stiffness matrix and the particular choices of the functions $\mathbf{F}_{T}$ and $S(t)$ constructed above. These solutions are distinct since:

$$
\forall m \in \mathbb{N}, \quad \mathbf{R}_{T}^{a}\left(\frac{1}{\beta^{m}}\right)=(0,0), \quad R_{T}^{b}\left(\frac{1}{\beta^{m}}\right)=\left(\frac{1}{\beta^{m}} \frac{e}{1-e^{2}}, 0\right)
$$

Using this result and proposition 1, one may easily construct two distinct solutions of problem $\mathcal{P}_{3}$ with the particular form of Eq. (46) of the stiffness matrix. Let $U_{N}^{a}(t), U_{N}^{b}(t), \mathbf{U}_{T}^{a}(t), \mathbf{U}_{T}^{b}(t)$, $R_{N}^{a}(t), R_{N}^{b}(t)$ and $F_{N}(t)$ be the functions defined on ]0,1[ by: 


$$
\begin{aligned}
U_{N}^{a}(t) & =U_{N}^{b}(t)=0, \\
\mathbf{U}_{T}^{a}(t) & =\mathbf{R}_{T}^{a}+\mathbf{F}_{T}, \\
\mathbf{U}_{T}^{b}(t) & =\mathbf{R}_{T}^{b}+\mathbf{F}_{T}, \\
R_{N}^{a}(t) & =R_{N}^{b}(t)=-\frac{1}{\mu} S(t), \\
F_{N}(t) & =\frac{1}{\mu} S(t)+e F_{T 1}(t),
\end{aligned}
$$

where $F_{T 1}(t)$ is the first component of $\mathbf{F}_{T}(t)$. Defining $\mathbf{F}(t)$ by $\mathbf{F}(t)=F_{N}(t) \mathbf{e}_{N}+F_{T 1}(t) \mathbf{e}_{T 1}+$ $F_{T 2}(t) \mathbf{e}_{T 2}$ and $\mathbf{U}^{a}, \mathbf{U}^{b}, \mathbf{R}^{a}, \mathbf{R}^{b}$, similarly, one gets two distinct solutions in $W^{1, \infty}\left(0,1 ; \mathbb{R}^{3}\right)$ of problem $\mathcal{P}_{3}$ with the particular form of Eq. (46) of the stiffness matrix. These two solutions exist for any value of the friction coefficient in the interval $] 0,1 / e[$.

Therefore, the solution of a quasi-static Signorini problem with Coulomb friction is not unique, in general, at least in the functional framework $W^{1, \infty}(0, T)$, [and therefore in $\left.W^{1, p}(0, T)\right]$.

\section{References}

[1] A. Signorini, Rend. di Matematica, Roma 18 (1959) 1-45.

[2] G. Fichera, Mem. Accad. Naz. Lincei, Ser. 87 (1964) 91-140.

[3] J.L. Lions, G. Stampacchia, Comm Pure Appl Math 20 (1967) 493-519.

[4] G. Duvaut, J.L. Lions, Les Inéquations en Mécanique et en Physique. Dunod, Paris, 1972.

[5] D. Kinderlehrer, G. Stampacchia, An Introduction to Variational Inequalites and Their Applications. Academic Press, New York, 1980.

[6] C. Baiocchi, A. Capelo, Variational and Quasivariational Inequalities. Wiley, New York, 1984.

[7] G. Duvaut, J.L. Lions, Journal de Mécanique 10 (1971) 409-420.

[8] G. Duvaut, C. R. Acad. Sci. Paris Série A 290 (1980) 263-265.

[9] J.T. Oden, E.B. Pires, ASME J Appl Mech 50 (1983) 67-76.

[10] M. Cocu, Int. J. Engng Sci. 22 (1984) 567-575.

[11] J. Jarušek, Czec. Mat. J. 33 (1983) 237-261.

[12] J. Jarušek, Czec. Mat. J. 34 (1984) 619-629.

[13] A. Klarbring, Eur J Mech A/Solids 9 (1990) 53-85.

[14] M. Cocu, E. Pratt, M. Raous, Int. J. Engng Sci. 34 (1996) 783-798.

[15] A. Klarbring, Ingenieur-Archiv 60 (1990) 529-541.

[16] J.J. Moreau, in G. Capriz, G. Stampacchia (Eds.), New Variational Techniques in Mathematical Physics, Edizioni Cremonese, Roma, 1973, pp. 173-322.

[17] Marques M.D.P. Monteiro, Differential Inclusions in Nonsmooth Mechanical Problems. Birkhaüser, Basel, Boston, Berlin, 1993.

[18] J.J. Moreau, J Differential Equations 26 (1977) 347-374. 